\title{
On the Application of DOA Estimation Techniques to UMTS System
}

\author{
E. Del Re , L. Pierucci, S. Marapodi \\ Department of Electronics and Telecommunications \\ V.S. Marta, 350139 FIRENZE - ITALY \\ piencci@lenst.det.unifi.it
}

\begin{abstract}
The paper describes the application of multiple signal classification (MUSIC) and its derivative algorithms to the estimation of direction of arrival (DOA) of multipath signals received from multiple users on the uplink of a wideband direct sequence code division multiple access (wideband DS-CDMA) system. Due to the multiple-access interference (MAI) and multipath, a high number of signals impinges on the antenna array and this scenario causes conventional direction-finding algorithms (such as MUSIC) fail. The proposed approach extends the use of MUSIC and allows to provide unique estimates even when the number of DOA's exceeds the number of antenna array elements.
\end{abstract}

\section{INTRODUCTION}

The next generation mobile communication systems, called Universal Mobile Teleconmunication System (UMTS) must support wideband services with quality as fixed network.

UMTS adopts Direct Sequence CDMA access scheme to improve bandwidth efficiency, all users communicate simultaneously in the same band and hence, MAI in addition to multipath significantly degrades performance. In UMTS system moreover, data rates may vary widely and high rate users may produce severe MAI in the uplink signals.

The use of antenna array in the CDMA system can improve system capacify, quality and reduce severe MAI from high rate users also by recovering DOA's information.

The DOA acknowledge is usefut to direct in the down link the main lobe of the transmitting antenna to cover only the interested users, reducing the interference towards the other users and allowing a lower level of electromagnetic pollution that it is the main goal.

The DOA methods using the eigenvectors decomposition are based on the assumption that the number of antennas is greater than the number of impinging signals (users and replicas) and these algorithms work fine only when the desired signal is orthogonal to the interference signals. In C.DMA system each user is identified by an unique code orthogonal to the other user codes. The scrambling codes proposed in the $3^{\text {rd }}$ Generation Partnership Project (3GPP) standards have not sufficient orthogonality properties to guarantee a good users recovering and the multipath channel contributes towards to degrade the codes properties. The interference of other users remains on the desired user decreasing performance and conventional MUSIC algorithm fails.

The novelty of this approach is to implement for each user a DOA estimator (MUSIC or IterativeMUSIC) for each replica. The estimators work in parallel at the output of matched filters, one for each path.

In this case only one peak of MUSIC spectrum is estimated at a time, providing a high reliability in DOA estimate values and it is more independent from the effect of multiple paths even if the complexity increases.

This innovative approach of Music algorithm overcomes the intrinsic limits of standard Music and it is able to estimate correctly the DOA of all users and of all replicas with a number of array elements much less than the total number of spatially separated signals impinging on the antenna array. In this paper, we first describe the model of signals received at antenna array, then a brief review of Music and Iterative-Music algorithms is provided. Finally the simulation results of innovative approach for DOA estimation are shown according the $3 \mathrm{GPP}$ standards.

\section{SIGNAL MODEL}

The received signal in the uplink of a DS-CDMA system at an antenna array of P-elements in the base station can be described: 


$$
x(n)=\sum_{k=1}^{\kappa} u_{k}(n)+n(n)
$$

$\mathrm{x}(\mathrm{n})$ is the $\mathrm{n}$-th sample of Px1 array output vector, $\mathrm{n}(\mathrm{n})$ is AWGN with covariance matrix $\sigma^{2} I$ and $I$ is the matrix identity;

$\mathrm{u}_{\mathrm{k}}(\mathrm{n})$ is the contribution of the $\mathrm{k}$-th users, considering $\mathrm{K}$ active users randomly distributed around the base station

$$
u_{k}(n)=\sum_{i=1\}}^{M} \sum_{i=1}^{l} c_{k l}(i) d_{k}(i) s_{k}\left(n-i T-\tau_{k l}\right) a_{k l}
$$

where $L$ is the number of paths

$M$ is the number of transmitted symbols,

$d_{k}$ (i) i-th symbol transmitted by the k-th user,

$c_{k l}(i) c o m p l e x$ attenuation of the $i-t h$ symbol of the

$k$-th user over the 1 -th path

$\mathrm{T}$ is the symbol interval

$\tau_{k l}$ the delay of $k$-th user over the 1-th path,

$\mathrm{s}_{\mathrm{kl}}$ short spreading code of $\mathrm{k}$-th user over the 1-th path.

For uniform linear array (ULA) we can consider the steering vector

$$
a_{k l}=\left[e^{-i \phi_{1, k l}}, e^{-j \phi_{2, k i}}, \ldots e^{-j \phi_{F, k i}}\right]^{T}
$$

where:

$\phi_{p, k t}=(p-1) 2 \pi r \sin \theta_{k l} / \lambda$

$\theta_{k}$ is the DOA of $k$-th user over $l$-th path

$r$ is the interelement distance

$\lambda$ is the wavelength

According to 3GPP standard only the scrambling code can discriminate the desired user and correlating $P$ received signals for each user and each path, the received signal after code-matched filters is considering 1 -th path and $\mathrm{k}$-th user:

$$
y_{k l}(i)=\sum s_{k}^{*}\left(n-i T-\tau_{k l}\right) x(n)
$$

After the correlation for each user using signals already compensated of delay of multipaths, we can obtain in output of the matched filter a despreaded signal independent by the other users (if the codes orthogonal properties are good) containing the DOA information for each path of the desired user.

Now it is possible to use DOA algorithms (such as Music) considering the covariance matrix at the output of matched filter:

$$
R_{y: y}=A \quad D \quad A^{H}+\sigma^{2} l
$$

where $D$ is the covariance matrix of the received signal and the matrix A contains the column steering vectors for the $\mathrm{L}$ replicas.

\section{DOA ESTIMATION}

MUSIC algorithm (Multiple Signal Classification)

It is very simple and it is based on eigenstructure method [2].

Considering multipath delays and complex coefficients of the channel known, Music estimator can work at the output of matched filters on a number of signals equal to the number of paths of the desired user.

The number of channel replicas (L) in real case, is less than the number of antenna elements and the correlation matrix $\mathrm{R}_{\mathrm{yy}}$ can be eigendecomposed.

The eigenvectors associated to the (P-L) smaller eigenvalues spanned the noise subspace and are orthogonal to the signal subspace containing the $\mathrm{L}$ directions information.

The search for $\mathrm{L}$ directions is made by looking for peaks in the MUSIC spectrum given by

$$
P_{M U}(\theta)=\frac{1}{\sum\left|s_{\theta}^{H} v_{i}\right|^{2}}
$$

where $\underline{s}_{t}$ is the frequency scanning vector $v_{i}$ is the eigenvector associated to the (P-L) smallest eigenvalues of $R_{y y^{\prime}}$.

The peaks of the Music's power spectrum provide the true DOA values. The main drawback of this algorithm is the complexity due mainly to Singular Value Decomposition. to calculate the eigenvalues according to $6 * N^{3}$ (where $N$ is the number of considered bits to calculate the covariance matrix) [2].

\section{Iterative Music (I-Music)}

The modified Music algorithm based on the Incremental Multi-parameter (IMP) algorithm [3] iteratively removes the desired signal from the data, then searching on the residue for further signals. The algorithm converges to a maximum likelihood solution for the considered number of signals.

The Iterative-Music is also used at the output of the matched filter on the covariance matrix $\mathrm{R}_{y y^{\prime}}$. First, the I-Music calculates the spectrum 


$$
P\left(\vartheta_{i}\right)=\frac{a^{H}\left(\vartheta_{i}\right) R_{y: y} a\left(\vartheta_{i}\right)}{a^{H}\left(\vartheta_{i}\right) a\left(\vartheta_{i}\right)}, i=\left[-90^{\circ}-90^{\circ}\right]
$$

The position of main peak of this 'pseudo-spectrum' estimates the angular location of the desired user. Then subsequent steps of the algorithm remove the estimated peak and iteratively search the other ones and related angular position [3]. I-Music does not require a Singular Value Decomposition and the computational complexity is much less expensive than Music.

\section{THE NEW APPROACH AND RESULTS}

Due to the insufficient orthogonality properties of the 3GPP scrambling codes and to multipath effects, the interference from other users remains on the desired user decreasing MUSIC algorithm performance when simultaneously estimates the DOAs of all the replicas of the desired user. Considering to analyse the selected user at the output of matched-filters, our approach proposes to use one DOA estimator for each path of selected user. In this case only one peak of MUSIC spectrum is estimated at a time, providing a high reliability in DOA estimate values and it is more independent from the effect of multiple paths even if the complexity increases. This innovative approach of Music algorithm overcomes the intrinsic limits of standard Music and it is able to estimate correctly the DOA of all users and of all replicas with a number of array elements much less than the total number of spatially separated signals impinging on the antenna array as shown in the following results.

The major radio link parameters are used according 3GPP standards and listed in Table 1.

\begin{tabular}{|c|c|}
\hline \multicolumn{2}{|c|}{ Radio link parameters } \\
\hline Bandwidtli & $5 \mathrm{MHz}$ \\
\hline Carrier frequency & $\sim 2 \mathrm{GHz}$ (uplink) \\
\hline Spreading Factor & 4.256 \\
\hline Information bit rate & $960 \mathrm{~Kb} / \mathrm{s}$ to $30 \mathrm{~Kb} / \mathrm{s}$ \\
\hline Spreading code & $\begin{array}{l}\text { OVSF (Orthogonal Variable } \\
\text { Spreading Factor) } \\
\text { Long Scrambling Code: } \\
\text { Gold codes } \\
\text { Short Scrambling Code: } \\
\text { S(2) Tamily }\end{array}$ \\
\hline Modulation & QPSK \\
\hline
\end{tabular}

Table 1 - Radio link parameters.
A spatial and temporal channel has been implemented, enclosing multipath fading and Doppler spread effects, the direction of arrival for each path and each user and antenna array geometry. The propagation channel is modelled as a sum of resolvable three paths with a time delay difference between adjacent paths such that it is more than a chip length and attenuation according Table 2.

\begin{tabular}{|c|c|c|c|}
\hline PATH & $\begin{array}{l}\text { AMPLITUDE } \\
(\mathrm{dB})\end{array}$ & $\begin{array}{l}\text { DELAY } \\
(\mu s)\end{array}$ & $\begin{array}{l}\text { DOPPLER } \\
\text { SPECTRUM }\end{array}$ \\
\hline 1 & 0 & 0 & Rayleigh \\
\hline 2 & -2 & 0.26 & Rayleigh \\
\hline 3 & -6 & 0.52 & Rayleigh \\
\hline
\end{tabular}

Table 2 - Channel model

An uniform linear antenna array with element's separation of half a carrier wavelength and 8 elements is considered.

We assume that the receiver knows the spreading codes, multipath delays and the channel complex coefficients.

At the antenna receiver in each matched filter the received signal is multiplied by the spreading code with recovered path time delays.

In all simulation the energy per bit-to-background noise ratio $\left(\mathrm{E}_{\mathrm{b}} / \mathrm{N}_{n}\right)$ for the desired user is set to $8 \mathrm{~dB}$ and the following load configurations are used: 16 users configuration for a global rate of $3.51 \mathrm{Mb} / \mathrm{s} ; 64$ users configuration for a global rate of $3.78 \mathrm{Mb} / \mathrm{s}$.

The DOA estimator is reused for each channel path: therefore in our case, three DOA estimators work in parallel to provide the DOA information for each desired user and for each replica. Each estimator searches for only one signal of arrival and therefore its estimate is more accurate.

The performance of DOA estimation is less sensitive to the number of incoming signals. Simulation results show an accurate DOA estimation even when the number of users increases .

The I-Music presents very closed performance respect to the Music algorithm as shown in Fig.1-2$3-4$. 


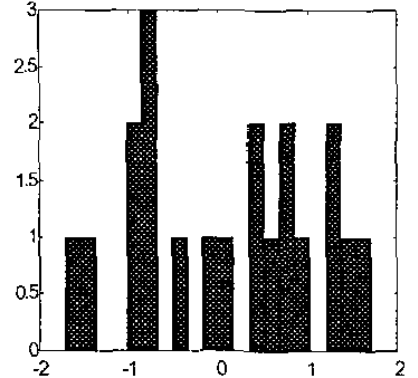

Path 1-DOA $=54.6^{\circ} \mathrm{MSE}=1.03$

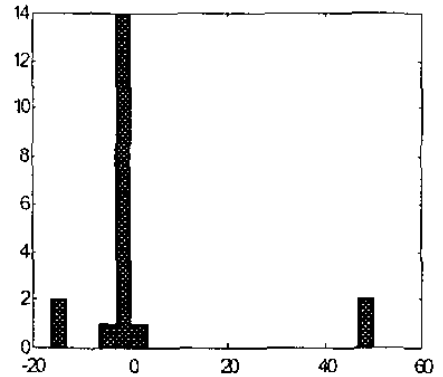

Path 2-DOA $=8.7^{\circ} \mathrm{MSE}=277.7$

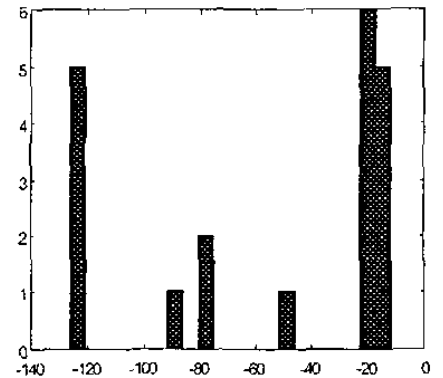

Path .3-DOA $=78.6^{\circ}$ MSE $=5170.1$

Fig1 - Music DOA estimation - Histogram of error estimation (degrees) around the DOA true value and Mean square error for three replicas of desired user in 16 users configuration
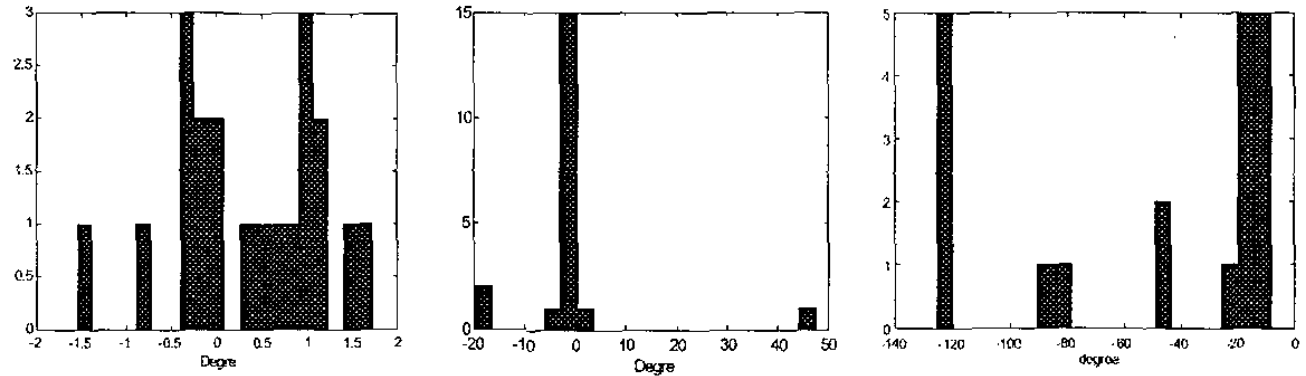

Path 1 - DOA $=54.6^{\circ} \mathrm{MSE}=0.78$ Path 2 - DOA $=8.7^{\circ} \mathrm{MSE}=150.06$ Path $3-\mathrm{DOA}=78.6^{\circ} \mathrm{MSE}=4917.0$

Fig.2 - I-Music DOA estimation - Histogram of error estimation around the DOA true value and Mean square error for three replicas of desired user and 16 users configuration
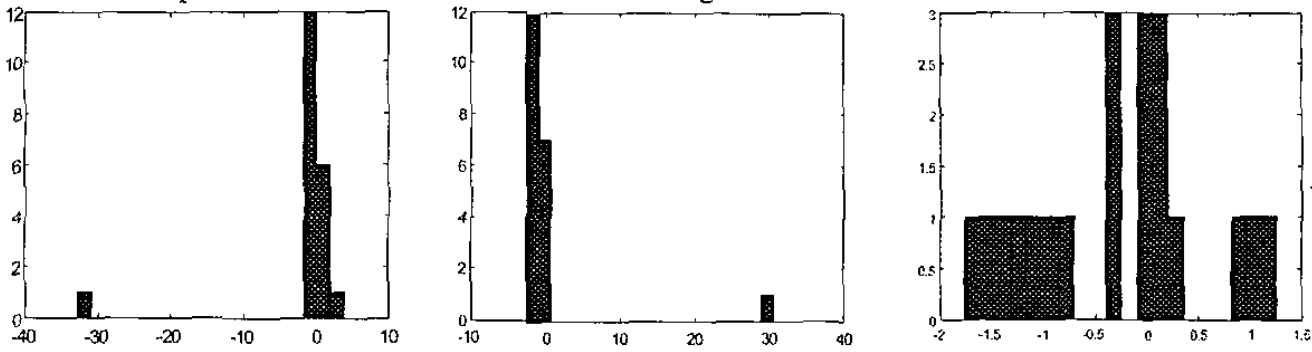

Path 1- DOA $=35.9^{\circ} \mathrm{MSE}=55.19$ Path 2-DOA $=30.6^{\circ} \mathrm{MSE}=48.04$

Path $3-\mathrm{DOA}=2.4^{\circ} \mathrm{MSE}=0.79$

Fig. 3 - Music DOA estimation - Histogran of error estimation around the DOA true value and Mean square error for three replicas of desired user and 64 users configuration

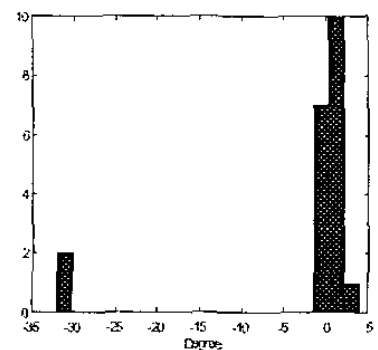

Path 1-DOA $=35.9^{\circ} \mathrm{MSE}=100.93$

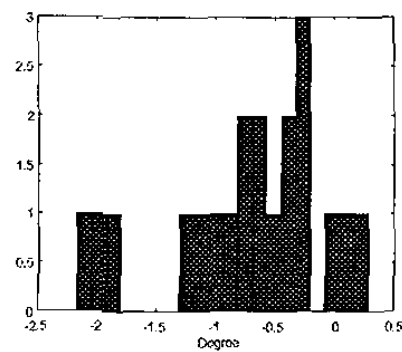

Path 2 - $\mathrm{DOA}=-30.6^{\circ} \mathrm{MSE}=0.98$

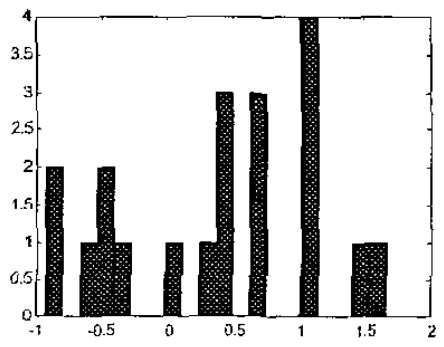

Path $3-\mathrm{DOA}=2.4^{\circ} \mathrm{MSE}=0.68$

Fig.4 - 1-Music DOA estimation - Histogram of error estimation around the DOA true value and Mean square error for three replicas of desired user and 64 users configuration 

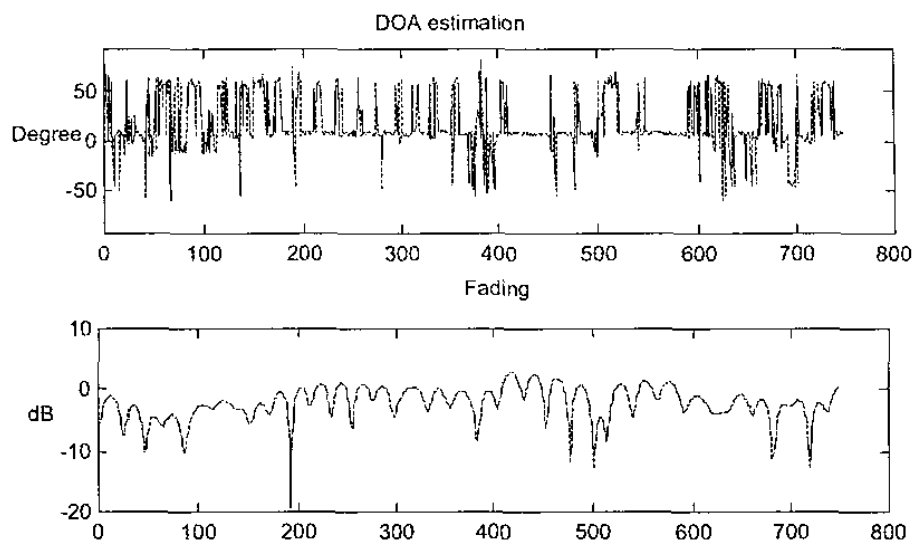

Fig.5 - I-Music DOA estimation for path 2 of desired user and fading propagation, 16 users configuration.

The results highlight a higher diffusion of error values in DOA estimation correlated to the channel attenuation more evident in the third replica.

The cases where both DOA's algorithms fail (Fig. 5), are strictly correlated with the fading therefore the DOA estimation could be improved deleting DOA punctual values lied with fading propagation in the estimation average.

\section{CONCLUSIONS}

In this paper, a new approach of MUSIC and Iterative-Music algorithms for DOA estimation in CDMA system was proposed, using MUSIC (or Iterative-Music) algorithm for each desired user and for each replica.

In this way, the algorithm accounts for the MAI arising from multipath propagation and asynchronous transmission, and is applicable in CDMA systems where the number of antenna array elements is often smaller than the number of desired DOAs.

The results highlight remarkable performance to estimate DOAs for all user and all paths, even in the case of maximum number of simultaneously users (full chip rate is $3.84 \mathrm{Mchips} / \mathrm{s}$, according the 3GPP standard).

This approach can be winner at the base station to estimate DOAs of uplink signals.

Much work remains to be done however, on analysing a different geometry of antenna array and on the calibrations needed to apply on the down-link the DOA values estimated in the uplink, to direct the main lobe of transmitter antenna towards the desired user and beams nulls towards the interfering ones.

\section{Acknowledgment}

The authors would like to thank Marconi Mobile to support this research.

\section{REFERENCES}

[1] 3GPP Standard: http://www.3gpp.org

[2] Haykin S., 1991, "Adaptive Filter Theory", Prentice-Hall International Ed.

[3] A. Morrison, B.S. Sharif, S. Sali, O.R. Hinton, 2000, "An Iterative DOA Algorithm For a Space-Time DS-CDMA Rake Receiver.", 3G Mobile Communication Technologies, Conf. Pub. No. 471 .

[4] Zhongding Lei, Teng Joon Lim, "Estimation of Directions of Arrival of Multipath signals in CDMA Systems", IEEE Trans. on Comm., Vol 48, No. 6, June 2000 